\title{
Automated Tomographic Reconstruction in the IMOD Software Package
}

\author{
David N. Mastronarde
}

Boulder Laboratory for 3-D Electron Microscopy of Cells, Dept. of MCD Biology, University of Colorado, Boulder, CO.

Automated processing of tilt series to generate tomographic reconstructions is an important goal for increasing throughput and reducing the effort needed to achieve good reconstructions. Recent developments in IMOD $[1,2]$ have made it possible to build high-quality tomograms automatically.

There are new IMOD programs that can pick a well-distributed set of gold fiducial markers for alignment of a tilt series. In typical operation, all gold beads are found on 3 projection views near zero tilt, then 3 independent runs of the Beadtrack program track these beads through 11 views around zero tilt. Various metrics from this tracking are combined to assign a score to each bead. The desired number of fiducials is then chosen to achieve as even a distribution as possible, drawing on the highest scoring beads first and avoiding beads that appear elongated or are situated too near other beads (Fig. 1A). When beads are on two surfaces, points are chosen to achieve a good distribution on both surfaces.

Sobel filtering can now be used for refining bead position during tracking. The Sobel filter, when applied to images where beads are scaled to an appropriate size, highlights the edges of the beads and allows a better estimate of the bead's center than cross-correlation or centroid-based positions, especially when there is other strong density underlying a part of the bead. As shown in Fig. 1B, the mean residual error from a fiducial alignment is typically reduced by $\sim 22 \%$ (from 0.65 to 0.51 pixels) for tilt series of plastic-embedded material. Use of the method with tilt series of frozen-hydrated samples also leads to lower errors, but the reduction is only $\sim 10 \%$ and more smoothing must be applied prior to the Sobel filter to obtain this benefit.

Tests with several data sets suggest that automatic seed finding, followed by tracking with Sobel filter centering, outperforms RAPTOR [3]. For example, the mean residual error for global alignment was 0.36 pixels from these methods vs. 0.68 pixels with RAPTOR for the cryo-tilt series in Fig 1B; and 0.35 vs. 0.76 pixels for the unbinned IMOD tutorial data set, available at http://bio3d.colorado.edu/imod.

Tiltalign, the program that computes fiducial alignment, can now use robust fitting, in which the weights of individual points are adjusted iteratively, based on their residual error in the fit. Typically, robust fitting gives a weight of 0 to $\sim 2 \%$ of points and weights of $0.01-0.5$ to another $\sim 3 \%$ of points. The alignment with aberrant points given low weights should be as good as one with their positions corrected by the user, provided there is a sufficient excess of points for alignment.

With these new capabilities, tilt series with fiducial markers can be reconstructed automatically and potentially have a better alignment than was obtained previously after making a seed model manually, 
tracking in Beadtrack, and correcting fiducial positions manually. A new program, Batchruntomo, can process a data set automatically, given a set of parameter settings in a text file. It can follow any of the pathways for alignment in the eTomo user interface and performs all processing steps through tomogram generation, except the step for setting angles and positions. It can also run optional steps such as CTF correction, or erasing of gold beads. A user interface for setting parameters to control batch processing of data sets is being developed. All programs are available in IMOD 4.6.18 and higher. [4]

\section{References:}

[1] J. R. Kremer et al., J. Struct. Biol. 116 (1996) p. 71.

[2] D. N. Mastronarde, J. Struct. Biol. 120 (1997) p. 343.

[3] F. Amat et al., J. Struct. Biol. 161 (2008) p. 260.

[4] This work was supported by NIH/NIGMS grant 8P41-GM103431 to Andreas Hoenger and by NIH/NIBIB grant 5R01-EB005027 to D. N. Mastronarde. Mary Morphew provided the tilt series shown in Fig. 1B.
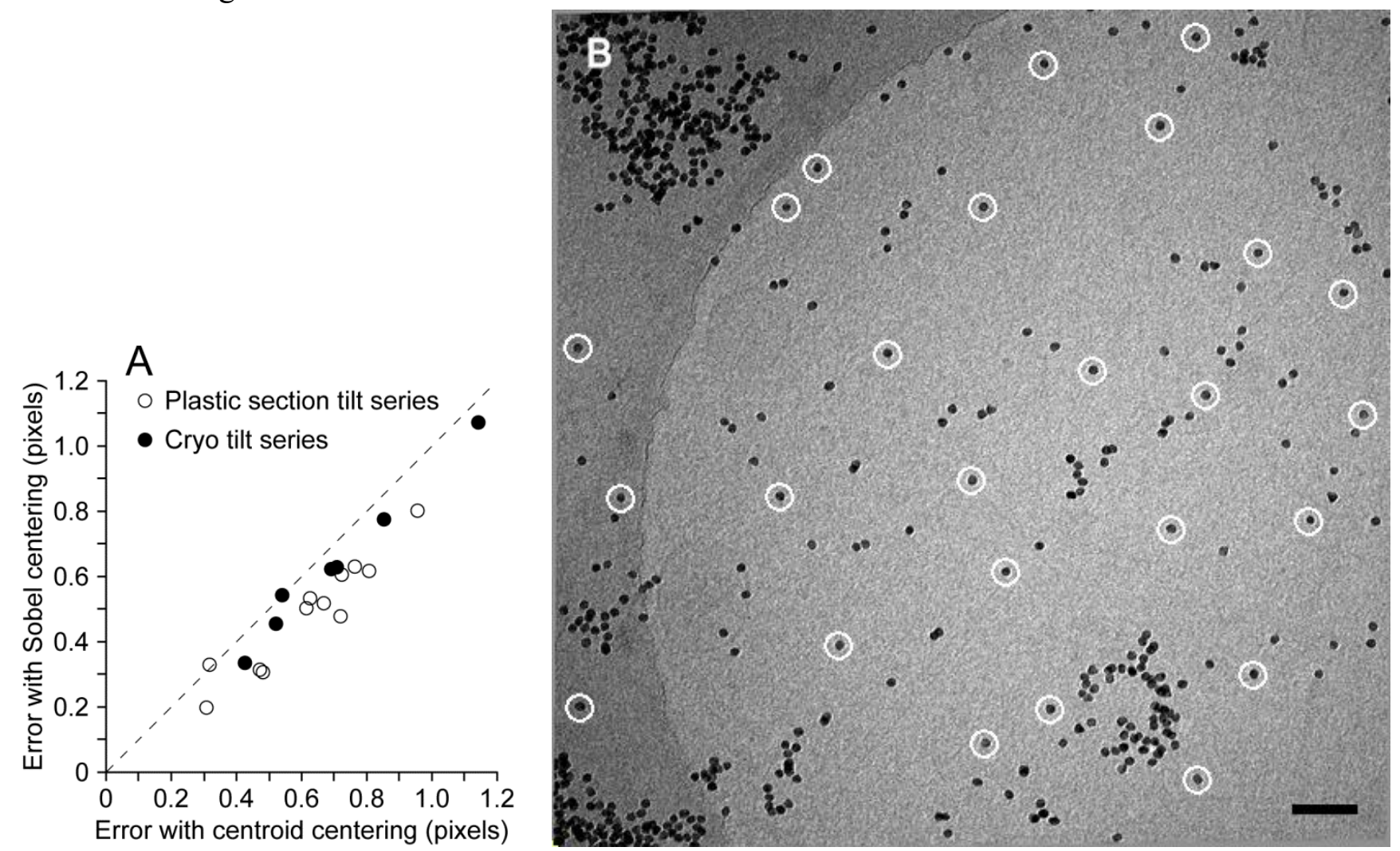

Figure 1. A) Mean residual error with the Sobel-filter centering plotted vs. error with the centering based on bead centroids, for data sets tracked in Beadtrack and aligned in Tiltalign with no correction of bead positions. The new centering produces $\sim 22 \%$ improvement for plastic section tilt series and $\sim 100 \%$ improvement for cryo-tilt series. B) Automatically chosen seed model of 25 points on the zerotilt view of a series from a frozen-hydrated cell infected with bovine papilloma virus. Beads in clumps and elongated beads have been successfully avoided. Scale bar is $100 \mathrm{~nm}$. 